\title{
Differential Patterns of Amygdala and Ventral Striatum Activation Predict Gender-Specific Changes in Sexual Risk Behavior
}

\author{
Elizabeth C. Victor, Alexandra A. Sansosti, Hilary C. Bowman, and Ahmad R. Hariri \\ Laboratory of NeuroGenetics, Department of Psychology and Neuroscience, Duke University, Durham, North Carolina 27708-0086
}

\begin{abstract}
Although the initiation of sexual behavior is common among adolescents and young adults, some individuals express this behavior in a manner that significantly increases their risk for negative outcomes including sexually transmitted infections. Based on accumulating evidence, we have hypothesized that increased sexual risk behavior reflects, in part, an imbalance between neural circuits mediating approach and avoidance in particular as manifest by relatively increased ventral striatum (VS) activity and relatively decreased amygdala activity. Here, we test our hypothesis using data from seventy 18- to 22-year-old university students participating in the Duke Neurogenetics Study. We found a significant three-way interaction between amygdala activation, VS activation, and gender predicting changes in the number of sexual partners over time. Although relatively increased VS activation predicted greater increases in sexual partners for both men and women, the effect in men was contingent on the presence of relatively decreased amygdala activation and the effect in women was contingent on the presence of relatively increased amygdala activation. These findings suggest unique gender differences in how complex interactions between neural circuit function contributing to approach and avoidance may be expressed as sexual risk behavior in young adults. As such, our findings have the potential to inform the development of novel, gender-specific strategies that may be more effective at curtailing sexual risk behavior.
\end{abstract}

Key words: amygdala; reward; sexual risk behavior; threat; ventral striatum; young adults

\section{Introduction}

Each year, $50 \%$ of new cases of sexually transmitted diseases are attributed to individuals between 15 and 24 years of age, a disproportionate number, as this age group accounts for only $25 \%$ of all sexually active individuals (CDC, 2011). Despite the obvious importance of better understanding factors that shape the emergence of such risky sexual behavior, relatively few studies have examined the contributions of individual differences in brain function to the expression of this risk. Identifying biological mechanisms of risk is important because it has the potential to not only direct the search for novel intervention targets but also inform strategies for prevention. Demos et al. (2012) reported that in young adult women the magnitude of activity in the ventral striatum (VS) to sexual images predicts an increase in new sexual partners 6 months later. This pattern is consistent with the critical role of the VS in coordinating information processing throughout a distributed corticostriatal circuit mediating ap-

Received Feb. 22, 2015; revised April 13, 2015; accepted May 5, 2015.

Author contributions: E.C.V. and A.R.H. designed research; E.C.V., A.A.S., and H.C.B. performed research; E.C.V., A.A.S., H.C.B., and A.R.H. analyzed data; E.C.V., A.A.S., H.C.B., and A.R.H. wrote the paper.

The Duke Neurogenetics Study was supported by Duke University and NIH Grant DA033369, and A.R.H. is supported by NIH Grants DA033369 and DA031579. We thank Spenser Radtke for assistance with subject recruitment and Annchen Knodt for assistance with the figures.

The authors declare no competing financial interests.

Correspondence should be addressed to Elizabeth Victor, Department of Psychology and Neuroscience, 417 Chapel Drive, Box 90086, Duke University Durham, NC 27708-0086. E-mail: elizabeth.victor@duke.edu.

DOI:10.1523/JNEUROSCI.0737-15.2015

Copyright $\odot 2015$ the authors $\quad 0270-6474 / 15 / 358896-05 \$ 15.00 / 0$ proach behaviors, reward drive, and impulsivity (Hariri, 2015), and provides initial support for the importance of variability in reward-related brain function in the expression of sexual risk behavior, as has been consistently reported for other risk behaviors including drug use (Koob and Volkow, 2010) and antisocial behaviors (Buckholtz et al., 2010).

However, limited research has investigated the neural mechanisms underlying the processing of both appetitive and aversive cues in humans (Bogdan and Pizzagalli, 2006; Ossewaarde et al., 2011; Park et al., 2011; Porcelli et al., 2012; Choi et al., 2014, 2015). Given that many health-risk behaviors occur in the context of both positive and negative cues (e.g., sex with a stranger could be interpreted as both exciting and dangerous), understanding how young adults evaluate situations simultaneously representing rewards and dangers may usefully inform prevention and intervention efforts.

Recent work from our laboratory has demonstrated that the influence of reward-related VS activity on risk behaviors may be modulated by threat-related activity of the amygdala, which as the hub of a distributed corticolimbic circuit mediates recognition and reaction to potential danger (Hariri, 2015). Specifically, we have found that the expression of stress-related problem drinking, a high-risk behavior, is most pronounced in young adults with a combination of relatively high reward-related VS activity and low threat-related amygdala activity (Nikolova and Hariri, 2012). Thus, the expression of risk-related behaviors may reflect the relative balance between neural circuits mediating approach and avoidance behaviors. 
Here we explore how amygdala activity may moderate the association between VS activity and the expression of sexual risk behavior over time in young adults. We hypothesized that consistent with the findings of Demos et al. (2012) relatively increased VS activity would predict an increase in the number of sexual partners over time. However, consistent with the findings of Nikolova and Hariri (2012), we further hypothesized that this VS-associated risk would only be expressed in the context of relatively decreased amygdala activity.

\section{Materials and Methods}

Overview of study design and participants. Beginning in May 2012, selfidentified heterosexual participants were recruited from the ongoing Duke Neurogenetics Study (DNS), which assesses a wide range of behavioral and biological traits, to complete an online assessment of sexual history. Any heterosexual participant having completed the DNS before May 2012 or anytime after was eligible. Therefore, some participants were enrolled immediately following their completion of the DNS, whereas others were enrolled years later $(M=188.72 \mathrm{~d}$, $S D=257.15$; range $=0 \mathrm{~d}-3.19$ years $)$. All participants $(N=70)$ provided informed consent in accordance with Duke University guidelines, and were between the ages of 18 and 22 years old $(M=19.93, S D=1.34)$. The sample included 46 females and 24 males. The self-identified racial/ethnic makeup was $47 \%$ European American $(n=33)$, 37\% Asian American $(n=26), 9 \%$ African American $(n=6), 7 \%(n=5)$ Latino, and $7 \%$ other $(n=5)$. Participants were paid $\$ 10$ for completion of a baseline assessment and every 3 months subsequently were e-mailed a follow-up survey in which they were asked to indicate how many new vaginal sexual partners they have had in the past 3 months. Participants were able to complete up to two follow-up surveys and were paid a \$5.00 Amazon gift card for each follow-up survey completed.

All participants were free of the following study exclusions: (1) medical diagnoses of cancer, stroke, diabetes requiring insulin treatment, chronic kidney or liver disease, or lifetime history of psychotic symptoms; (2) use of psychotropic, glucocorticoid, or hypolipedemic medication; and (3) conditions affecting cerebral blood flow and metabolism (e.g., hypertension). Diagnosis of any current DSM-IV Axis I disorder or select Axis II disorders (antisocial personality disorder and borderline personality disorder), assessed with the electronic Mini International Neuropsychiatric Interview (Sheehan et al., 1998) and Structured Clinical Interview for the DSM-IV subtests (First et al., 1996) were not an exclusion, as the DNS seeks to establish broad variability in multiple behavioral phenotypes related to psychopathology. No participants met criteria for a personality disorder and nine $(12.9 \%)$ participants from our final sample met criteria for at least one Axis I disorder (1 agoraphobia, 4 alcohol abuse, 1 substance abuse, 2 past major depressive episode, 1 social phobia). These individuals did not report significantly more sexual partners at follow-up compared with the participants without a DSM-IV diagnosis $\left(t_{(70)}=\right.$ $0.34, p=0.74)$. Sexual orientation was an additional exclusionary criterion only for this supplemental study.

$B O L D$ fMRI data acquisition. Each participant was scanned using a research-dedicated GE MR750 3T scanner equipped with high-power high-duty-cycle $50 \mathrm{mT} / \mathrm{m}$ gradients at $200 \mathrm{~T} / \mathrm{m} / \mathrm{s}$ slew rate, and an eightchannel head coil for parallel imaging at high bandwidth up to $1 \mathrm{MHz}$ at the Duke-UNC Brain Imaging and Analysis Center. A semiautomated high-order shimming program was used to ensure global field homogeneity. A series of 34 interleaved axial functional slices aligned with the anterior commissure-posterior commissure (AC-PC) plane were acquired for full-brain coverage using an inverse-spiral pulse sequence to reduce susceptibility artifact (TR/TE/flip angle $=2000 \mathrm{~ms} / 30 \mathrm{~ms} / 60$; $\mathrm{FOV}=240 \mathrm{~mm} ; 3.75 \times 3.75 \times 4 \mathrm{~mm}$ voxels; interslice skip $=0$ ). Four initial receptive field excitations were performed (and discarded) to achieve steady-state equilibrium. To allow for spatial registration of each participant's data to a standard coordinate system, highresolution three-dimensional structural images were acquired in 34 axial slices coplanar with the functional scans (TR/TE/flip angle $=7.7$ $\mathrm{s} / 3.0 \mathrm{~ms} / 12$; voxel size $=0.9 \times 0.9 \times 4 \mathrm{~mm}$; FOV $=240 \mathrm{~mm}$, interslice skip $=0)$.
$B O L D F M R I$ data preprocessing. Images for each subject were realigned to the first volume in the time series to correct for head motion, spatially normalized into a standard stereotactic space (Montreal Neurological Institute template) using a 12-parameter affine model (final resolution of functional images $=2 \mathrm{~mm}$ isotropic voxels), and smoothed to minimize noise and residual difference in gyral anatomy with a Gaussian filter, set at $6 \mathrm{~mm}$ full-width at half-maximum. Voxelwise signal intensities were ratio normalized to the whole-brain global mean. Variability in single-subject whole-brain functional volumes was determined using the Artifact Recognition Toolbox (http://www.nitrc.org/projects/artifact_detect). Individual whole-brain BOLD fMRI volumes meeting at least one of two criteria were flagged and regressed out when determining task-specific effects: (1) significant mean-volume signal intensity variation (i.e., within volume mean signal greater or $<4 \mathrm{SD}$ of mean signal of all volumes in time series), and (2) individual volumes where scan-to-scan movement exceeded $2 \mathrm{~mm}$ translation or $2^{\circ}$ rotation in any direction.

BOLD fMRI paradigms. Our fMRI paradigms have been described in detail previously (Nikolova and Hariri, 2012; Carré et al., 2013). Briefly, the amygdala paradigm consists of four task blocks requiring matching of emotional facial expressions interleaved with five control blocks of matching geometric shapes. During task blocks, participants view a trio of faces (with neutral, angry, fearful, or surprised expressions) and match one of two faces (bottom) identical to a target face (top). During control blocks, participants match simple geometric shapes. Here, we focus on general activity associated with all four expressions (Ahs et al., 2014) using the contrast of All Faces > Shapes.

Our VS paradigm consists of a number guessing task wherein participants receive predominantly positive feedback ( $80 \%$ correct guess), predominantly negative feedback (20\% correct guess), or no feedback. There are three pseudorandomly presented blocks of each condition. Participants are unaware of the fixed outcome probabilities associated with each block and are led to believe their performance will determine a net monetary gain at the end of the scanning session. Instead, all participants receive $\$ 10$. Here we focus on reward-related VS activity from the contrast of Positive $>$ Negative Feedback.

$B O L D$ fMRI data analysis. Individual contrast images for from the amygdala and VS paradigms were used in second-level random effects models accounting for scan-to-scan and participant-to-participant variability to determine mean condition-specific regional responses using one-sample $t$ tests. A combined statistical threshold of $p<0.05$, corrected across the amygdala and VS, and $\geq 10$ contiguous voxels was applied to the above contrasts. Our amygdala regions-of-interest were defined using the automatic anatomical labeling option in the Wake Forest University PickAtlas (Wake Forest University School of Medicine, Winston-Salem, NC). Our VS regions of interest were defined using a 10 $\mathrm{mm}$ sphere centered approximately: $x= \pm 12 y=12 z=-10$ (Nikolova and Hariri, 2012; Carré et al., 2013).

To facilitate regression analyses, BOLD parameter estimates were extracted from the peak activation voxel from amygdala and VS clusters exhibiting main effects of task using the VOI tool in SPM8. In addition to producing the necessary values for our regression models, extracting parameter estimates from functional clusters activated by our paradigms rather than clusters specifically correlated with our dependent and independent variables of interest eliminates the possibility of any correlation coefficient inflation that may result when an explanatory covariate is used to select a region of interest (Viviani, 2010).

Assessment of sexual behavior. Participants were asked to indicate how many lifetime vaginal sexual partners they have had, as well as their number of sexual partners over the past 3 months. Change scores were created based on the difference between participants' vaginal sexual partners at the latest follow-up minus their baseline number of partners to determine whether participants accumulated new partners and how many. A score of zero is equal to no new partners during the longitudinal data collection period. Participants completed an average of 1.48 follow-up assessments and the average time between baseline and the last follow-up measure was 11.08 months $(\mathrm{SD}=6.5$; range: $3.0-20.0$ months).

Data analyses. Follow-up reports of number of sexual partners were distributed non-normally (skewness $>0.57, \mathrm{SE}=0.29$, Kurtosis $>1.13$, 


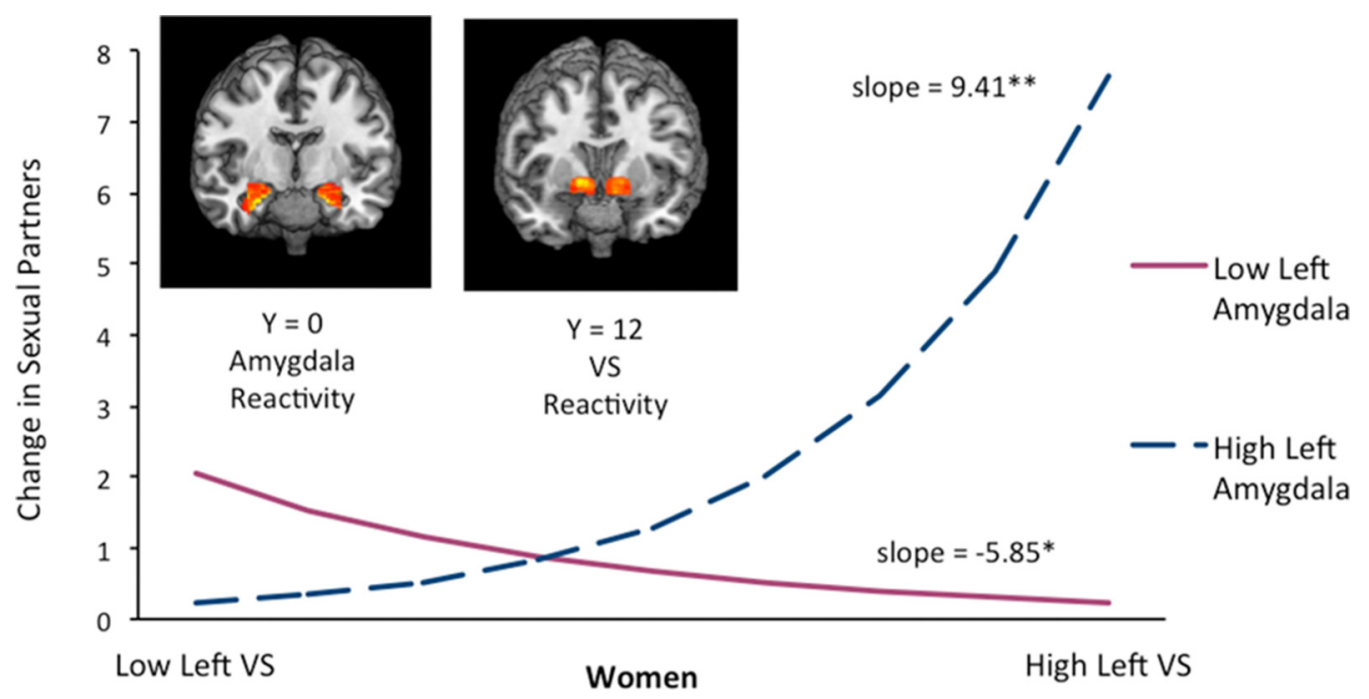

Figure 1. Statistical parametric map from the DNS parent sample illustrating mean bilateral amygdala activity (top, left; $N=811 ; x=28, y=-4, z=-20 ; t=44.1, p<0.000001, k_{\mathrm{E}}=$ 194) and mean bilateral VS activity (top, right; $\left.N=821 ; x=-12, y=8, z=-10 ; t=13.59, p<0.000001, k_{\mathrm{E}}=301\right)$. Activation clusters are overlaid onto a canonical structural brain image in the axial plane. Graphical representation of the interaction between LVS and Left Amygdala in women $\left[N=46\right.$; Wald $\left.\chi^{2}(5, N=46)=16.36, p=0.001\right]$. High reactivity $=1 S D$ above the mean; low reactivity $=1 \mathrm{SD}$ below the mean.

$\mathrm{SE}=0.57)$. We therefore tested our hypotheses using generalized linear modeling (GzLM) in SPSS 20.0. Interpretation of GzLM is similar to ordinary least-squares regression models, although a test of overall model fit is used in place of an $F$ test. For all dependent variables, we specified the Poisson distribution, which is appropriate for count data (i.e., non-negative integers) with positive skew.

We included gender, age, and mental health status (i.e., DSM-IV diagnosis) in all models to ensure that these factors did not confound the hypothesized relations. We also standardized all continuous predictor variables before analyses to ease interpretation of event rate ratios. Specifically, we estimated four separate GzLMs predicting change in number of vaginal partners over time. In each model, main effects were entered, as well as all two-way and three-way interactions with gender, amygdala activity, and VS activity. Only effects surviving Bonferroni correction for multiple comparisons $(p<0.006)$ were considered further.

\section{Results}

Sample demographics

The majority of the sample reported engaging in vaginal sex at least once in their lifetime $(n=42,60 \%)$. The mean number of vaginal sexual partners at baseline was 1.28 ( $\mathrm{SD}=0.68)$. The mean increase in vaginal sexual partners at the last follow-up was 0.71 (SD = 1.51). There were no significant differences between men and women in self-reported baseline or change in selfreported number of sexual partners $(t=0.05, p=0.96 ; t=1.02$, $p=0.31$, respectively). Although there was not a significant association between age and self-reported number of partners at baseline $(r=0.17, p=0.16)$, younger participants were more likely to report a greater increase in partners over time $(r=$ $-0.24, p=0.04)$. Notably, distribution analyses revealed two individuals with outlying values ( $>3$ SD from M; both subjects reported an increase in 8 partners between baseline and followup). Given the low rate of sexual risk behavior reported in the sample, these outliers were not excluded, as they likely best represent young adults engaging in sexual risk behavior.

\section{Main effects of task}

As expected, our fMRI paradigms elicited robust affect-related amygdala and reward-related VS activity across the entire parent sample of 917 participants (Fig. 1). In our substudy sample of 70 participants, there were no significant effects of gender $t_{(70)}$ val- ues $<0.88, p$ values $>0.17)$ or age $(r$ values $<0.22 ; p$ values $>$ $0.07)$ on VS or amygdala activity in either hemisphere.

\section{Poisson regressions}

We found a significant interaction between gender, left amygdala activity, and left VS activity $(b=44.07, p=0.002)$, as well as between gender, left amygdala activity, and right VS activity $(b=$ $37.91, p=0.001)$ predicting number of sexual partners over time (model of left amygdala, left VS, and gender: Wald $\chi^{2}(9, N=$ $70)=47.68, p=0.012$; model of left amygdala, right VS, and gender: Wald $\left.\chi^{2}(9, N=70)=52.10, p<0.001\right)$. In the models including right amygdala with right and left VS, no factors survived Bonferroni correction. Age and mental health status had no significant effect in any model, and were subsequently dropped from the within-gender analyses below.

To explore the differences in the relationships between amygdala and VS activity and sexual risk behavior between men and women we ran the significant models (Models 1 and 2) separately by gender. For women, interactions between left amygdala and left VS $(b=28.02, p=0.01)$ and left amygdala and right VS $(b=27.02, p=0.003)$ were significant in predicting number of partners accumulated over time. Women with both higher ( $>1$ SD above the mean) VS and higher (1 SD above the mean) amygdala activity showed the greatest accumulation of partners over time (Fig. 1). For men, the interactions between left amygdala and left VS $(b=4.46, p=0.03)$ and the main effect of right VS $(b=13.11, p<0.001)$ were significantly associated with greater increases in sexual partners over time. Among men with high ( $>1$ SD above the mean) left VS activity and low $(<1 \mathrm{SD}$ below the mean) left amygdala activity, there was the greatest accumulation of partners over time (Fig. 2). The two-way interaction was trending toward significance in the left amygdala and right VS model among men $(b=3.81, p=0.051)$.

\section{Discussion}

Our current results are broadly consistent with those of Demos et al. (2012) demonstrating that the magnitude of VS activity is associated with sexual risk behaviors in the form of increased partners over time. Our results usefully extend this prior work by 


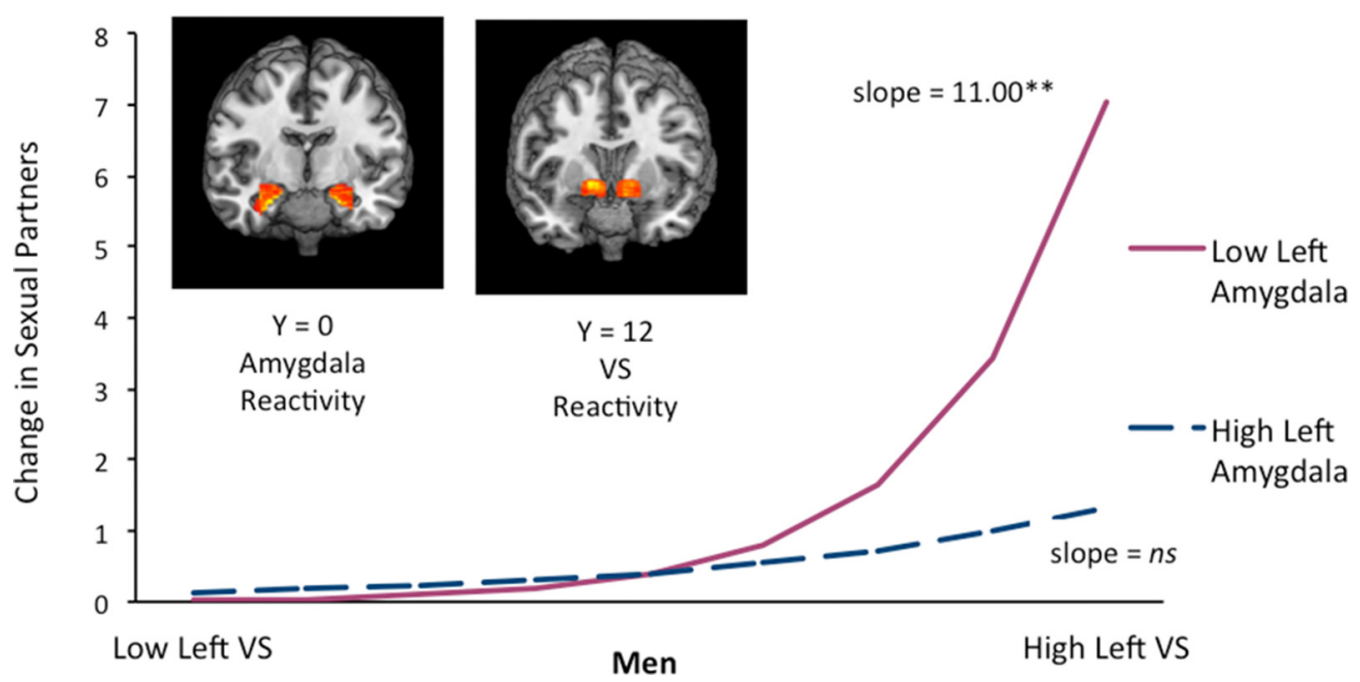

Figure 2. Statistical parametric map from the DNS parent sample illustrating mean bilateral amygdala activity (top, left; $N=811 ; x=28, y=-4, z=-20 ; t=44.1, p<0.000001, k_{\mathrm{E}}=$ 194) and mean bilateral VS activity (top, right; $N=821 ; x=-12, y=8, z=-10 ; t=13.59, p<0.000001, k_{\mathrm{E}}=301$ ). Activation clusters are overlaid onto a canonical structural brain image in the axial plane. Graphical representation of the interaction between LVS and Left Amygdala in men [graph; $N=24 ;$ Wald $\left.\chi^{2}(5, N=24)=16.40, p=0.001\right]$. High reactivity $=1$ SD above the mean; low reactivity $=1$ SD below the mean.

implicating relatively increased VS activity associated with more general, nonsexual cues in predicting risk that is expressed not only in women but also in men. Furthermore, our results suggest that the expression of VS-associated risk is moderated by the magnitude of amygdala activity in a gender-specific manner. Specifically, in men but not women relatively increased VS activity was associated with greater number of partners over time, only in the context of relatively decreased amygdala activity. This pattern is consistent with that previously reported for stress-related problem drinking (Nikolova and Hariri, 2012). In contrast, relatively increased VS activity in women was associated with greater partners over time only in the context of relatively increased amygdala activity.

The nature of these unexpected gender differences is unclear and warrants further consideration. We speculate that the observed patterns may reflect the greater importance of gender differences in amygdala-mediated physiologic arousal associated with the interaction of potential approach and avoidance cues present when young people make sexual decisions (e.g., decision to engage in sexual behavior with a new partner could reflect excitement about new sexual experiences, but also fear of potential STIs, unplanned pregnancy, or even violence). In women, such increased physiologic arousal may unmask the expression of risk behaviors associated with relatively increased VS activity. In men, however, increased amygdala activity may dampen the expression of this same VS-associated risk.

Given that our measure of amygdala activity (i.e., contrast of All Faces $>$ Shapes) is not specific to threat-related expressions (e.g., fear and anger) but rather more broadly salient social stim$\mathrm{uli}$, it is also possible that the increased activity associated with greater numbers of sexual partners among women is reflective of the amygdala's role as a critical relay of sensory cues associated with positive and negative outcomes. It is also possible that a third variable (such as a trait-level personality variable like impulsivity or reward sensitivity, or a situational variable, such as recent life stress) may be moderating or mediating the relationships between neural function and sexual behavior differently in men and women. For instance, Choi et al. (2015) found that individual differences linked to reward and threat was related to behavior such that for participants low in trait-level rewardsensitivity, the relationship between anxiety and behavior in a working memory task under threat of shock was positive, suggesting a greater motivational salience of threat to reward. In contrast, for participants high in trait-reward sensitivity, there was no relationship between anxiety and behavior, suggesting that these individuals are motivated by reward regardless of threatening cues. Future studies assessing gender differences in contemporaneous changes in peripheral physiology associated with amygdala activity and larger samples affording opportunity to evaluate moderating and mediating effects of trait personality and state affect would be useful in evaluating these hypotheses.

Although our results represent important extensions of a currently limited body of research regarding the neural mechanisms of real-world sexual risk behavior, they are not without limitations. First, although our sample is demographically representative of the larger university population from which it was drawn, our participants did not exhibit high levels of sexual risk behaviors (i.e., fewer that 2 partners lifetime), and therefore did not reflect nationally representative college samples, which typically report more than two partners in the past year (American College Health Association, 2013). Future studies specifically targeting young adults who engage in multiple sexual risk behaviors (e.g., poor condom use, drug and alcohol use, sex with strangers, etc.) will more accurately capture the extent to which variability in VS and amygdala activity predicts actual negative outcomes (e.g., STIs, unplanned pregnancies).

Second, our assessment of sexual activity was limited to selfreport, which may be affected by recall and selection bias. For instance, because many sexual behaviors may have occurred in the context of alcohol and drug use, accurate recall may be particularly challenging. In addition, given people's tendency to present themselves in a favorable light, a social desirability bias may have impacted both baseline and follow-up self-reports of sexual behavior.

Third, we did not measure VS and amygdala activity in response to sexually provocative stimuli but rather to more general representations of reward and affective arousal. It is possible that variability in VS and amygdala activity to such explicit stimuli 
may have different or nonexistent gender-specific patterns that may or may not map onto sexual risk behaviors. Moreover, the functional balance between the amygdala and VS reflects, in part, the regulatory influence of prefrontal circuits supporting executive control (Ernst et al., 2006) and a recent study has linked relatively decreased prefrontal activity during an inhibitory control task with increased sexual risk behaviors (Goldenberg et al., 2013). It will be interesting for future work to evaluate the functional dynamics between the VS, amygdala, and prefrontal cortex in response to sexually explicit scenarios and stimuli. These limitations notwithstanding, our current results suggest the importance of considering gender-specific patterns of interactions between functional neural circuits supporting approach and avoidance in the expression of sexual risk behavior in young adults.

\section{References}

Ahs F, Davis CF, Gorka AX, Hariri AR (2014) Feature-based representations of emotional facial expressions in the human amygdala. Soc Cogn Affect Neurosci 9:1372-1378. CrossRef Medline

American College Health Association (2013) National college health assessment: reference group executive summary spring 2013. Baltimore, MD: American College Health Association.

Bogdan R, Pizzagalli DA (2006) Acute stress reduces reward responsiveness: implications for depression. Biol Psychiatry 60:1147-1154. CrossRef Medline

Buckholtz JW, Treadway MT, Cowan RL, Woodward ND, Benning SD, Li R, Ansari MS, Baldwin RM, Schwartzman AN, Shelby ES, Smith CE, Cole D, Kessler RM, Zald DH (2010) Mesolimbic dopamine reward system hypersensitivity in individuals with psychopathic traits. Nat Neurosci 13: 419-421. CrossRef Medline

Carré JM, Hyde LW, Neumann CS, Viding E, Hariri AR (2013) The neural signatures of distinct psychopathic traits. Soc Neurosci 8:122-135. CrossRef Medline

Centers for Disease Control and Prevention (CDC) (2011) youth risk behavior surveillance: United states, 2010. Atlanta, GA: National Center for Health Statistics.

Choi JM, Padmala S, Spechler P, Pessoa L (2014) Pervasive competition between threat and reward in the brain. Scan 9:737-750. CrossRef Medline

Choi JM, Padmala S, Pessoa L (2015) Counteracting effect of threat on reward enhancements during working memory. Cogn Emot. Advance online publication. Retrieved on May 13, 2015. doi:10.1080/ 02699931.2014.993596. CrossRef Medline

Demos KE, Heatherton TF, Kelley WM (2012) Individual differences in nucleus accumbens activity to food and sexual images predict weight gain and sexual behavior. J Neurosci 32:5549-5552. CrossRef Medline

Ernst M, Pine DS, Hardin M (2006) Triadic model of the neurobiology of motivated behavior in adolescence. Psychol Med 36:299-312. CrossRef Medline

First M, Spitzer R, Gibbon M, William J (1996) Structured clinical interview schedule for DSM-IV axis 1 disorders, research version, patient/nonpatient edition. New York: Biometrics Research New York State Psychiatric Institute.

Goldenberg D, Telzer EH, Lieberman MD, Fuligni A, Galván A (2013) Neural mechanisms of impulse control in sexually risky adolescents. Dev Cogn Neurosci 6:23-29. CrossRef Medline

Hariri AR (2015) Looking inside the disordered brain. Sunderland, MA: Sinauer.

Koob GF, Volkow ND (2010) Neurocircuitry of addiction. Neuropsychopharmacology 35:217-238. CrossRef Medline

Nikolova YS, Hariri AR (2012) Neural responses to threat and reward interact to predict stress-related problem drinking: a novel protective role of the amygdala. Biol Mood Anxiety Disord 2:19. CrossRef Medline

Ossewaarde L, Qin S, Van Marle HJ, van Wingen GA, Fernández G, Hermans EJ (2011) Stress-induced reduction in reward-related prefrontal cortex function. Neuroimage 55:345-352. CrossRef Medline

Park SQ, Kahnt T, Rieskamp J, Heekeren HR (2011) Neurobiology of value integration: when value impacts valuation. J Neurosci 31:9307-9314. CrossRef Medline

Porcelli AJ, Lewis AH, Delgado MR (2012) Acute stress influences neural circuits of reward processing. Front Neurosci 6:157. CrossRef Medline

Sheehan DV, Lecrubier Y, Sheehan KH, Amorim P, Janavs J, Weiller E, Hergueta T, Baker R, Dunbar GC (1998) The mini-international neuropsychiatric interview (M. I. N. I.): the development and validation of a structured diagnostic psychiatric interview for DSM-IV and ICD-10. J Clin Psychiatry 59:22-33. Medline

Viviani R (2010) Unbiased ROI selection in neuroimaging studies of individual differences. Neuroimage 50:184-189. CrossRef Medline 